\title{
AMS-02 antiprotons are consistent with a secondary astrophysical origin
}

\author{
Mathieu Boudaud ${ }^{* a}$, Yoann Génolini ${ }^{b}$, Laurent Derome ${ }^{c}$, Juien Lavalle ${ }^{d}$, David \\ Maurin $^{c}$, Pierre Salati $^{e}$ and Pasquale D. Serpico ${ }^{e}$ \\ ${ }^{a}$ LPTHE, CNRS \& Sorbonne Université, 4 Place Jussieu, F-75005 Paris - France \\ ${ }^{b}$ Service de Physique Théorique, ULB, Boulevard du Triomphe, CP225, 1050 Brussels, Belgium \\ ${ }^{c}$ LPSC, Univ. Grenoble Alpes, CNRS, 53 avenue des Martyrs, F-38000 Grenoble, France \\ ${ }^{d}$ LUPM, UM \& CNRS, Place Eugène Bataillon, F-34095 Montpellier, France \\ ${ }^{e}$ LAPTh, USMB \& CNRS, 9 Chemin de Bellevue, F-7494 Annecy-le-Vieux, France \\ E-mail: boudaud@lpthe.jussieu.fr
}

The AMS-02 experiment has ushered cosmic-ray physics into precision era. Using the USINE code and an improved methodology, we extract the cosmic-ray transport parameters from the $\mathrm{B} / \mathrm{C}$ data. We then derive a robust range of predictions for the secondary antiprotons flux, accounting for several sources of uncertainties and their correlations. Combined with a correlation matrix of systematic errors for the antiprotons data, we show that the latter are consistent with a secondary origin. We present key elements relevant to dark matter search in this channel, notably by pointing out the inherent difficulties in achieving predictions at the percent-level precision.

36th International Cosmic Ray Conference -ICRC2019-

July 24th - August 1st, 2019

Madison, WI, U.S.A.

${ }^{*}$ Speaker. 


\section{Introduction}

The AMS-02 experiment has recorded several tens of thousands of $\bar{p}$ 's up to $\mathrm{TeV}$ energies [1]. For the first time, the measurement is dominated by systematic uncertainties (at a few percent level), implying new challenges for their interpretation. In Ref. [2] we addressed this issue, going beyond previous analyses of preliminary AMS-02 $\bar{p}$ 's data (e.g., [3]). We underline that CR $\bar{p}$ 's are one of the most sensitive astroparticle probes of annihilating/decaying DM in the GeV-TeV range, and any constraint on DM candidates depends on how well the astrophysical background is controlled. This is especially important as claims for $\bar{p}$ excesses attributed to DM are being debated $[4,5,6,7,8]$

\section{Methodology}

The flux of CR $\bar{p}$ 's at Earth depends on (i) the cross sections entering their production, scattering, and annihilation, (ii) the CR propagation model, (iii) the IS spectrum of the (most abundant) CR nuclei, and (iv) modulation of fluxes in the Solar cavity.

We make use of the parametrisation proposed in Ref. [9] (Param II) for the Lorentz invariant cross section of prompt $\bar{p}$ 's produced in $p p$ interactions. For nucleon-nucleon interactions, we use the scaling relation B proposed in Ref. [9], which provides the best agreement with LHCb data. We use the covariance matrices of errors on the parameters to propagate the uncertainties to the $\bar{p}$ flux calculation. Antineutrons ( $\bar{n}$ 's) and antihyperons are produced in hadronic interactions and decay into $\bar{p}$ 's. We include their contribution following Ref. [10]. The ratio between the total $\left(\sigma_{\text {inv }}^{\text {tot }}\right)$ and the prompt $\left(\sigma_{\text {inv }}\right) \bar{p}$ production cross section is shown in the left panel of Fig. 1 . In the case of vanishing non-prompt contribution and isospin violation, one would expect a constant value equal to 2 . Hence, not only the $\bar{p}$ flux receives an upwards correction of $\sim 20 \%$ to $50 \%$, but also acquires peculiar energy-dependent features, further affected by relatively large uncertainties. Inelastic annihilating and non-annihilating interactions of $\bar{p}$ 's with the IS medium (ISM) are treated following the procedure described in [11].

We use the 1D diffusion model implemented in USINE v3.5 [12] which assumes a thick diffusion halo size $L$, and a thin disc containing the sources and the gas. We use the most generic transport model defined in [13] (called BIG) where the transport parameters are fitted on B/C following the methodology described in [14], i.e. a model of the covariance matrix of B/C AMS-02 errors has also been incorporated in the fit. We compute the best-fit values of the free parameters (transport and nuisance parameters) and their covariance matrix. We fully propagate the transport uncertainty to the $\bar{p}$ flux from the covariance matrix.

Important inputs for the $\bar{p}$ calculation are the IS fluxes for the progenitors. We follow and extend the two-step procedure detailed in [13] (see Sec. III therein): we start from the best-fit parameters obtained from the $\mathrm{B} / \mathrm{C}$ analysis (BIG) and then perform a simultaneous fit of $\mathrm{H}$ [15], $\mathrm{He}, \mathrm{C}$, and O [16] AMS-02 data to determine the source parameters, i.e. four normalisations $\left({ }^{1} \mathrm{H}\right.$, ${ }^{4} \mathrm{He},{ }^{12} \mathrm{C}$, and $\left.{ }^{16} \mathrm{O}\right)$ and three slopes $\left(\alpha_{\mathrm{H}}, \alpha_{\mathrm{He}}\right.$, and a universal source slope $\alpha_{\mathrm{Z}>2}$ for all other species). We fit at the same time the high-rigidity diffusion break parameters which are better constrained by elemental fluxes than by the $\mathrm{B} / \mathrm{C}$ [13]. We include in the fit the covariance matrices of errors on $\mathrm{H}, \mathrm{He}, \mathrm{C}$, and $\mathrm{O}$ data (see App. C of [2]). The outputs of this fitting procedure are 
presented in Fig. 2. Note that we fix the Fisk potential $\phi_{\mathrm{FF}}$ for $\mathrm{H}, \mathrm{He}, \mathrm{C}$, and $\mathrm{O}$ data to the value yielded by the $\mathrm{B} / \mathrm{C}$ data fit [13]. While published AMS-02 data are from the same time period for $\mathrm{B} / \mathrm{C}, \mathrm{He}, \mathrm{C}$, and $\mathrm{O}(05 / 2011$ to $05 / 2016)$, they originate from a shorter period for $\mathrm{H}(05 / 2011$ to $11 / 2013$ ), so that the associated modulation level should be slightly different. However, as the fit is restricted to data above the $\bar{p}$ production threshold $\left(E_{k / n}>6 m_{p}\right)$, the impact is feeble, and in any case negligible compared to other uncertainties. Assuming the same modulation for $\bar{p}$ and $\mathrm{H}, \mathrm{He}$, $\mathrm{C}$, and $\mathrm{O}$ actually already gives a satisfactory description at low rigidity. For this reason, we did not include further uncertainty on $\phi_{\mathrm{FF}}^{\bar{p}}$. If anything, this would slightly enlarge the uncertainty of the prediction at low rigidity, improving further the consistency between our calculated $\bar{p}$ flux and the data.

In the context of the $\mathrm{B} / \mathrm{C}$ analysis, we stressed the importance of using a realistic covariance matrix of the data errors to avoid misleading conclusions [14]. We anticipate that the same is true for $\bar{p}$ 's. However, since this matrix is not directly provided by the AMS-02 collaboration, we build it from the published systematic errors and associated description of their physics origin [1], as shown in the right panel of Fig. 1. The covariance matrix associated with these systematics is then built based on a choice of their correlation length, $\ell$. We take as educated guesses for the correlations lengths (in unit of energy decade) $\ell_{\text {Acc. }}=0.1$ (acceptance), $\ell_{\text {Cut }}=1.0$ (rigidity cut-off), $\ell_{\text {Scale }}=4.0$ (rigidity scale), $\ell_{\text {Templ. }}=0.5$ (template fitting), $\ell_{\mathrm{XS}}=1.0$ (cross sections), $\ell_{\text {Unf. }}=1.0$ (unfolding), and $\ell_{\text {Sel. }}=0.5$ (selection). More details on this procedure are given in App. C of [2].
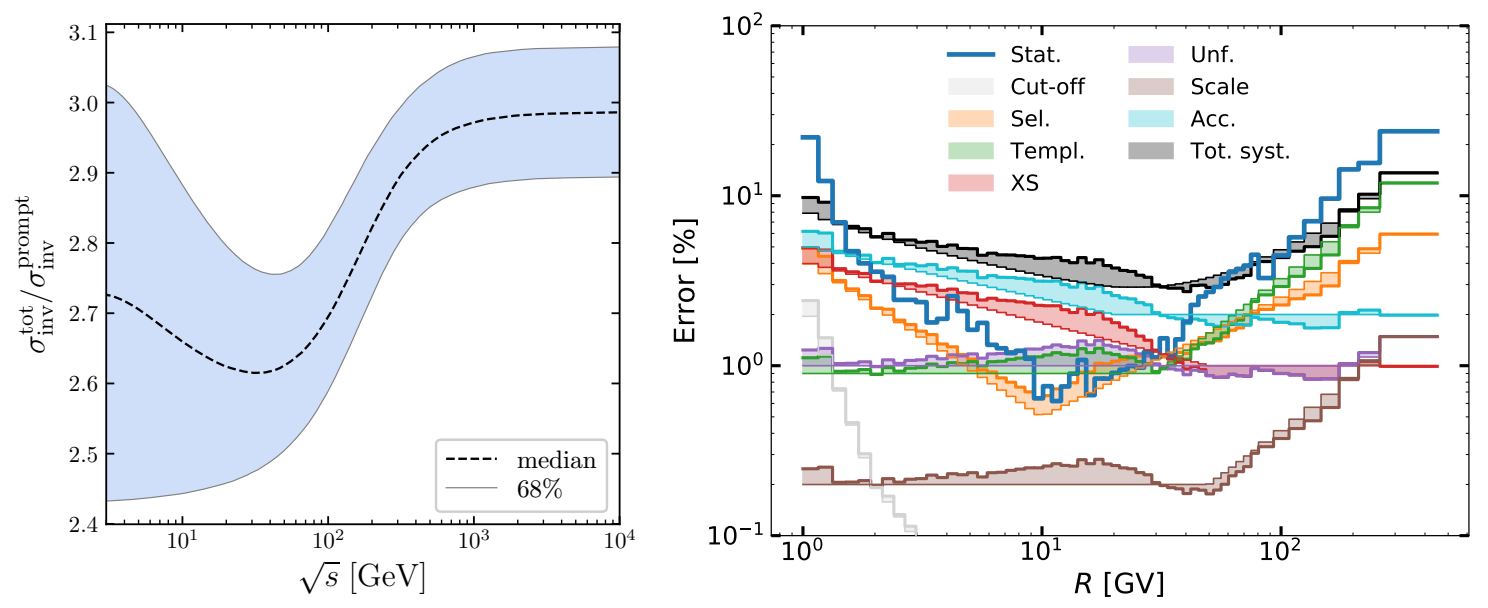

Figure 1: Left: ratio between total (including antihyperons and antineutrons) production cross section and promptly produced $\bar{p}$ cross section. Right: AMS-02 errors for $\bar{p}$ data. Statistical (Stat.) and Total Systematic (Tot. syst.) lines correspond to the errors provided in [17]. Individual contributions in the systematic errors namely rigidity cut-off (Cut-off), selection (Sel.), template fitting (Templ.), cross sections (XS), unfolding (Unf.), rigidity scale (Scale) and acceptance (Acc.), built from information provided in [1] are shown (coloured lines) before (thin) and after (thick) the rescaling applied to match the total systematic error. 


\section{Prediction of the $\bar{p}$ flux}

The left panel of Fig. 3 shows our baseline $\bar{p}$ flux prediction (not a fit) obtained from the bestfit values for the $\bar{p}$ production cross sections, the transport (BIG) and the associated parents fluxes, compared with AMS-02 data with errors taken as the quadratic sum of systematic and statistical errors (black crosses). The 'standard' residuals with respect to the baseline model are displayed in the middle panel. Note that the points do not include the model uncertainties, nor correlations in the data uncertainties. We also show on the same plot the $68 \%$ total confidence band for the model (grey band). This band could release the tension with the data, even before accounting for the information on the correlations in rigidity bins. The respective contributions of parents, cross sections and transport are also plotted. At tens of GV, the errors from transport and cross sections are almost constant and close to $10 \%$. At larger rigidities, the errors from transport and parents increase because of the increasing experimental uncertainty in the $\mathrm{B} / \mathrm{C}$ ratio and parent fluxes, respectively. At low rigidity, the error from transport grows for the same reasons and encompasses the uncertainty in the prediction of the low-rigidity behaviour (see App. E of [2]). However, we remind the reader that a visual comparison can be deceiving: the presence of non-diagonal values in the covariance matrices is responsible for a better agreement between the model and data than perceived in the residuals (see Table 1).

To test the actual compatibility of our prediction with the $\bar{p}$ data, we present two statistical tests which boil down to probabilistic statements in terms of $p$-value (see App. D of [2]). First, we propose a $\chi^{2}$ test, with the help of a covariance matrix of errors on both data and model:

$$
\chi^{2}=(\text { data }- \text { model })^{\mathrm{T}}\left(\mathscr{C}^{\text {model }}+\mathscr{C}^{\text {data }}\right)^{-1}(\text { data }- \text { model }) .
$$

The covariance matrices of the data $\mathscr{C}^{\text {data }}$ and the model $\mathscr{C}^{\text {model }}$ are given by the sum of the different contributions previously detailed (see App. B1 and App. C of of [2]). We find $\chi^{2} \approx 44$, and identifying the number of degrees of freedom (dof) with the number of $\bar{p}$ data points (57), we infer a corresponding $p$-value of 0.9 which is reported in the last line of Table. 1.

Such a test does not directly assess a possible overestimate of the errors, and also relies on the notion of number of dof (which may be a shaky concept in some circumstances, see e.g. the discussion in [18]). Thus, we also perform a Kolmogorov-Smirnov (KS) test, which obviates the above limitations. We compute the distribution of the 'eigen residuals' (z-score) corresponding to the residuals of the eigen vectors (data-model) of the total covariance matrix. In the bottom panel of Fig. 3 we show these 'eigen residuals' as a function of 'rigidity' (actually, the one rotated in the eigen basis, see App. D of [2]), and in the inset, we compare the corresponding histogram with a Gaussian. The KS test leads to a $p$-value of 0.27 which is also very good and is clearly consistent with the hypothesis that $\bar{p}$ 's are of secondary origin. For completeness, we also report in Table. 1 the $p$-values when considering different combinations of errors: (i) If there was no uncertainty in our baseline model, the covariance matrix of data errors alone $\left(\mathscr{C}^{\text {data }}\right)$ would already give enough freedom to allow for a very good agreement between the data and the secondary flux prediction; (ii) Considering only the statistical uncertainties in the data and the uncertainties in the model $\left(\sigma_{\text {stat }}\right.$ and $\mathscr{C}^{\text {model }}$ ), this prediction is marginally consistent with the data at the $2 \sigma$ level, with the KS test leading to an even better $p$-value. Also note the relevance of the KS test (as opposed to the $\chi^{2}$ test) to spot error overestimates, in the case of $\sigma_{\text {tot }}$ and $\mathscr{C}^{\text {model }}$; (iii) In the most realistic case 
considering both $\mathscr{C}^{\text {data }}$ and $\mathscr{C}^{\text {model }}, p$-values are acceptable for both the $\chi^{2}$ and KS test. Thus, not only is a secondary origin for the locally measured $\bar{p}$ 's statistically consistent with the data, but, as shown by these considerations, it is also robust with respect to error mismodelling in either model or data errors.

Table 1: Respective $p$-values for different sources of errors. We take dof $=57$, i.e. the number of $\bar{p}$ data. Total errors on data are defined to be $\sigma_{\text {tot }}=\sqrt{\sigma_{\text {stat }}^{2}+\sigma_{\text {syst }}^{2}}$.

\begin{tabular}{cccc}
\hline \hline Error considered & $\chi^{2} /$ dof & p-value $\left(\chi^{2}\right)$ & p-value $(\mathrm{KS})$ \\
\hline \hline$\sigma_{\text {stat }}$ & 23 & 0 & 0 \\
$\sigma_{\text {tot }}$ & 1.69 & $8.3 \times 10^{-4}$ & 0 \\
$\mathscr{C}^{\text {data }}$ & 0.84 & 0.79 & 0.98 \\
$\sigma_{\text {stat }}$ and $\mathscr{C}^{\text {model }}$ & 1.32 & 0.05 & 0.99 \\
$\sigma_{\text {tot }}$ and $\mathscr{C}^{\text {model }}$ & 0.37 & 1.0 & 0.04 \\
$\mathscr{C}^{\text {data }}$ and $\mathscr{C}^{\text {model }}$ & 0.77 & 0.90 & 0.27 \\
\hline
\end{tabular}
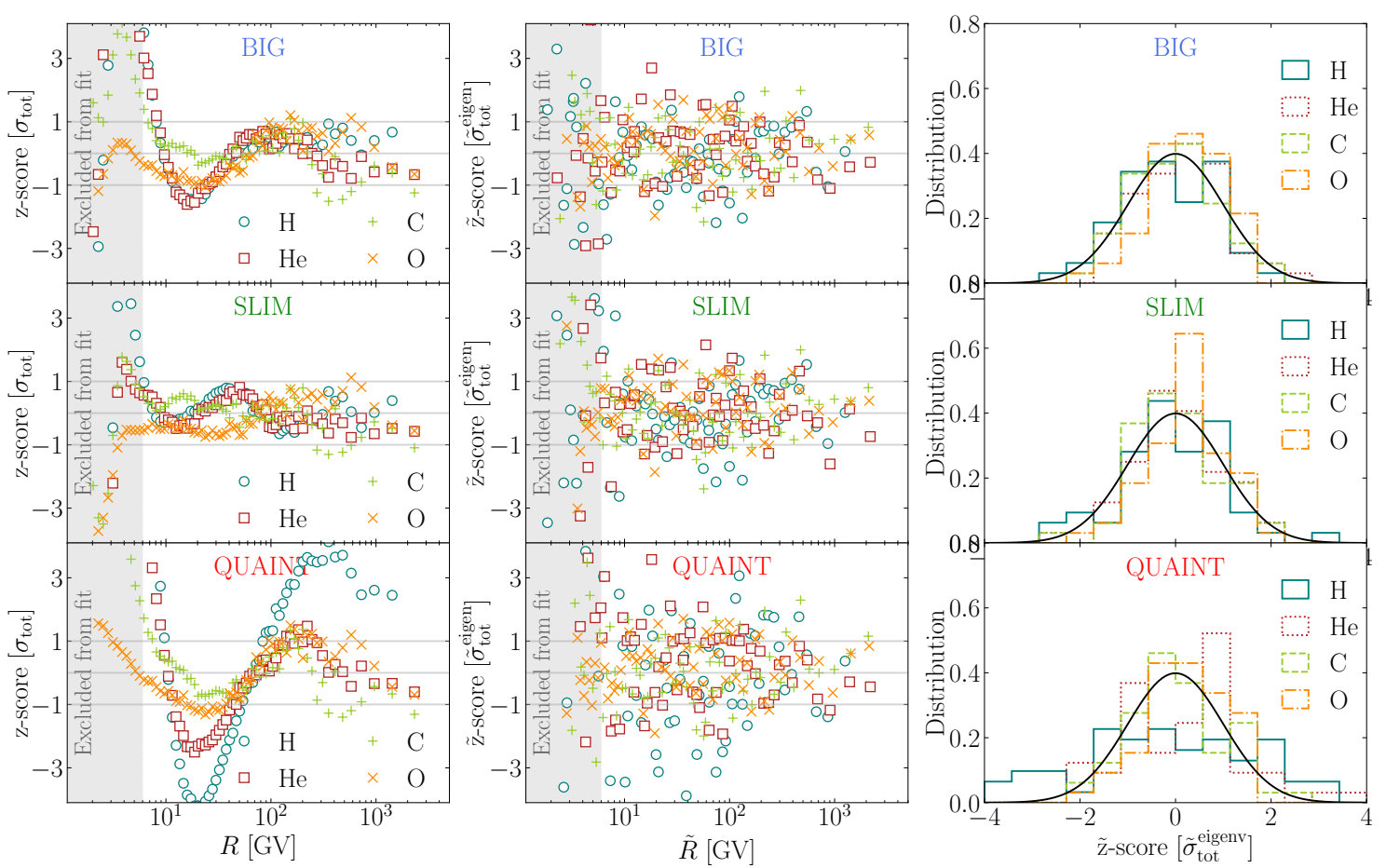

Figure 2: Z-score in the original basis (left) and in the eigenvector basis (centre), the distribution of the latter being shown as histograms (right). The rows correspond to the different propagation configurations introduced in [13], namely BIG, SLIM, and QUAINT (from top to bottom). In each panel, the element H, $\mathrm{He}, \mathrm{C}$, and $\mathrm{O}$ fit to AMS-02 data are symbol- and colour-coded. 

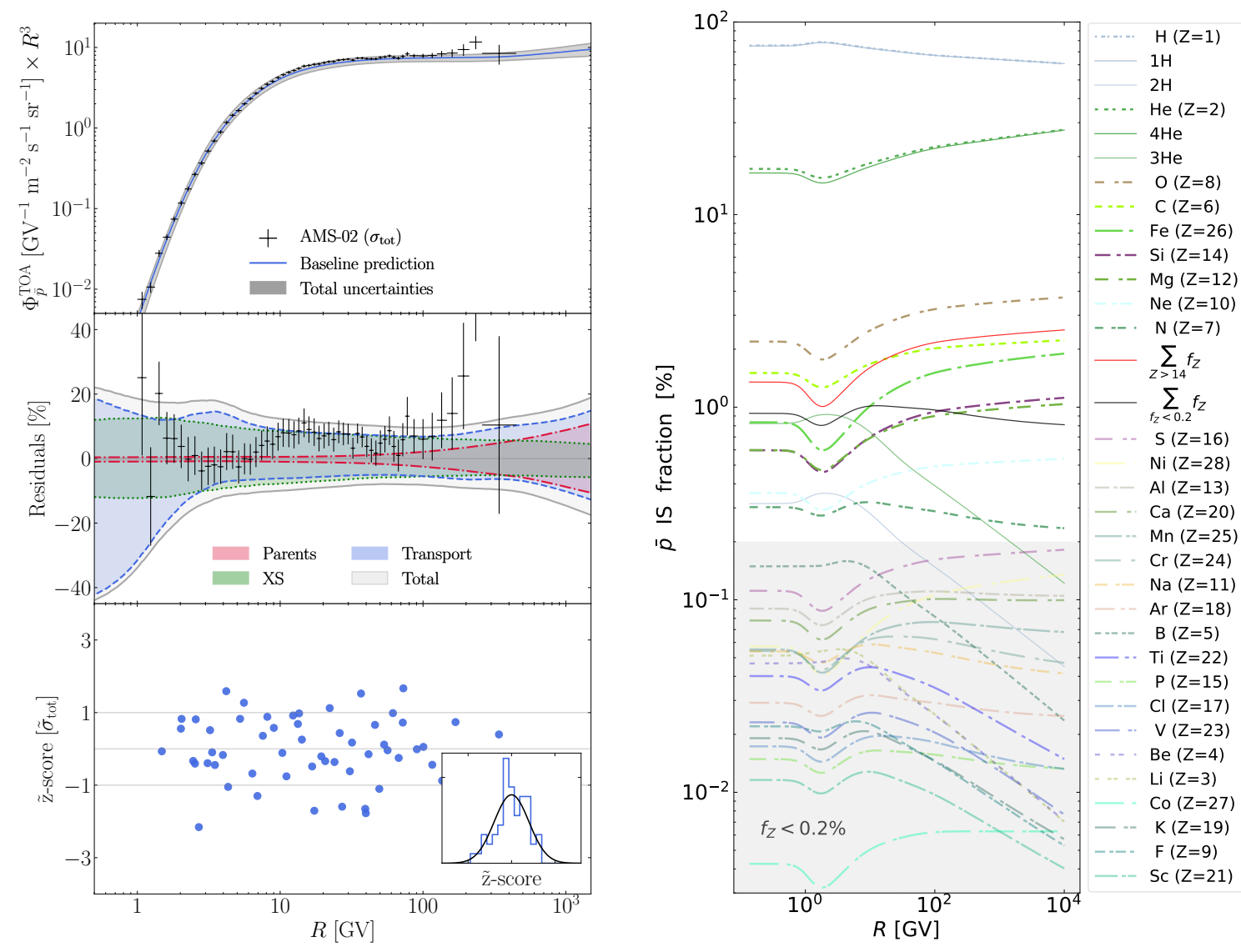

Figure 3: Left: comparison of $\bar{p}$ model and data (top panel), along with residuals and $68 \%$ total confidence interval for the model (grey) together with the transport (blue), the parents (red) and the cross sections (green) contributions (middle panel). The residuals of the eigen vectors of the total covariance matrix as well as their distribution are shown in the bottom panel and in the inset. Right: relative contributing fraction $f_{Z}$ (in percent) of $\bar{p}$ (thick dash-dotted lines) for propagation model BIG used in this study [13]. Specific contributions highlighted in thin solid lines: (i) broken-down isotopic contribution of ${ }^{1} \mathrm{H}$ and ${ }^{2} \mathrm{H}$ (blue), and ${ }^{3} \mathrm{He}$ and ${ }^{4} \mathrm{He}$ (green); (ii) contribution from all elements $Z>14$ (red); (iii) contribution from all elements whose fraction is $f_{Z}<0.2 \%$ (black). The grey area delimits the region within which contributions are below $0.2 \%$.

\section{Conclusion}

Percent-level details in the model predictions now matter, as do more subtle aspects of the data error treatment. We have presented a major upgrade of the $\bar{p}$ flux prediction and analysis by: (i) using the latest constraints on transport parameters from AMS-02 B/C data, (ii) propagating all uncertainties (with their correlations) on the predicted $\bar{p}$ flux, (iii) accounting for correlated errors in $\bar{p}$ data. With these novelties, we unambiguously show that the AMS-02 data are consistent with a pure secondary astrophysical origin. We stress that this conclusion is not based on a fit to the AMS-02 $\bar{p}$ data, but on a prediction of the $\bar{p}$ flux computed from external data. Our results should hold for any steady-stade propagation model of similar complexity, as they all amount to the same "effective grammage" crossed to produce boron nuclei (on which the analysis is calibrated), with 
roughly the same grammage entering the secondary $\bar{p}$ 's. More elaborate models would be less constrained and thus would make the agreement even better.

\section{References}

[1] M. Aguilar, L. Ali Cavasonza, B. Alpat, G. Ambrosi, L. Arruda, N. Attig et al., Antiproton Flux, Antiproton-to-Proton Flux Ratio, and Properties of Elementary Particle Fluxes in Primary Cosmic Rays Measured with the Alpha Magnetic Spectrometer on the International Space Station, Phys. Rev. Lett. 117 (2016) 091103.

[2] M. Boudaud, Y. Génolini, L. Derome, J. Lavalle, D. Maurin, P. Salati et al., AMS-02 antiprotons are consistent with a secondary astrophysical origin, arXiv e-prints (2019) [1906.07119].

[3] G. Giesen, M. Boudaud, Y. Génolini, V. Poulin, M. Cirelli, P. Salati et al., AMS-02 antiprotons, at last! Secondary astrophysical component and immediate implications for Dark Matter, JCAP 9 (2015) 23 [1504.04276].

[4] M.-Y. Cui, Q. Yuan, Y.-L. S. Tsai and Y.-Z. Fan, Possible Dark Matter Annihilation Signal in the AMS-02 Antiproton Data, Phys. Rev. Lett. 118 (2017) 191101 [1610.03840].

[5] A. Cuoco, M. Krämer and M. Korsmeier, Novel Dark Matter Constraints from Antiprotons in Light of AMS-02, Phys. Rev. Lett. 118 (2017) 191102 [1610.03071].

[6] I. Cholis, T. Linden and D. Hooper, A Robust Excess in the Cosmic-Ray Antiproton Spectrum: Implications for Annihilating Dark Matter, Phys. Rev. D99 (2019) 103026 [1903. 0254 9].

[7] A. Cuoco, J. Heisig, L. Klamt, M. Korsmeier and M. Kramer, Scrutinizing the evidence for dark matter in cosmic-ray antiprotons, Phys. Rev. D99 (2019) 103014 [1903.01472].

[8] S.-J. Lin, X.-J. Bi and P.-F. Yin, Investigating the dark matter signal in the cosmic ray antiproton flux with the machine learning method, arXiv e-prints (2019) [1903.09545].

[9] M. Korsmeier, F. Donato and M. Di Mauro, Production cross sections of cosmic antiprotons in the light of new data from the NA61 and LHCb experiments, Phys. Rev. D 97 (2018) 103019 [1802.03030].

[10] M. W. Winkler, Cosmic ray antiprotons at high energies, JCAP 2 (2017) 048 [1701. 04866 ].

[11] M. Boudaud, M. Cirelli, G. Giesen and P. Salati, A fussy revisitation of antiprotons as a tool for Dark Matter searches, JCAP 1505 (2015) 013 [1412.5696].

[12] D. Maurin, USINE: semi-analytical models for Galactic cosmic-ray propagation, arXiv e-prints (2018) [1807.02968].

[13] Y. Génolini, M. Boudaud, P. Ivo Batista, S. Caroff, L. Derome, J. Lavalle et al., Cosmic-ray transport from AMS-02 B/C data: benchmark models and interpretation, arXiv e-prints (2019) [1904.08917].

[14] L. Derome, D. Maurin, P. Salati, M. Boudaud, Y. Génolini and P. Kunzé, Fitting B/C cosmic-ray data in the AMS-02 era: a cookbook, arXiv e-prints (2019) [1904.08210].

[15] M. Aguilar, D. Aisa, B. Alpat, A. Alvino, G. Ambrosi, K. Andeen et al., Precision Measurement of the Proton Flux in Primary Cosmic Rays from Rigidity 1 GV to 1.8 TV with the Alpha Magnetic Spectrometer on the International Space Station, Phys. Rev. Lett. 114 (2015a) 171103. 
[16] M. Aguilar, L. Ali Cavasonza, B. Alpat, G. Ambrosi, L. Arruda, N. Attig et al., Observation of the Identical Rigidity Dependence of He, C, and O Cosmic Rays at High Rigidities by the Alpha Magnetic Spectrometer on the International Space Station, Phys. Rev. Lett. 119 (2017) 251101.

[17] M. Aguilar, L. Ali Cavasonza, G. Ambrosi, L. Arruda, N. Attig, S. Aupetit et al., Observation of New Properties of Secondary Cosmic Rays Lithium, Beryllium, and Boron by the Alpha Magnetic Spectrometer on the International Space Station, Phys. Rev. Lett. 120 (2018) 021101.

[18] R. Andrae, T. Schulze-Hartung and P. Melchior, Dos and don'ts of reduced chi-squared, arXiv e-prints (2010) [1012.3754]. 